\title{
Pi2-pulsations observed in energetic electron precipitation and magnetic field in association with a substorm surge
}

\author{
A. Åsnes ${ }^{1}$, J. Stadsnes ${ }^{1}$, J. Bjordal ${ }^{1}$, N. Østgaard ${ }^{2,3}$, D. L. Detrick ${ }^{4}$, T. J. Rosenberg ${ }^{4}$, and S. E. Haaland ${ }^{5,6}$ \\ ${ }^{1}$ Department of Physics, University of Bergen, Allegt. 55, 5007 Bergen, Norway \\ ${ }^{2}$ NASA/Goddard Space Flight Center, Greenbelt, MD 20771,USA \\ ${ }^{3}$ University of California, Berkeley, CA94720-7450, USA \\ ${ }^{4}$ University of Maryland, College Park, MD 20 742-24 331, USA \\ ${ }^{5}$ International Space Science Institute, Switzerland \\ ${ }^{6}$ Max-Planck Institut für extraterrestrische Physik (MPE), Garching, Germany
}

Received: 26 February 2003 - Revised: 12 January 2004 - Accepted: 9 February 2004 - Published: 14 June 2004

\begin{abstract}
For a substorm 24 July 1998 PIXIE observes the onset and expansion during a perigee pass of the Polar satellite. This gives an opportunity to follow the evolution of the onset and expansion phase, almost on a global scale with relatively high temporal resolution. The substorm is presented with multiple observations throughout the magnetosphere. Following the onset of the substorm we observe a localised region of modulated energetic electron fluxes following the passage of the westward travelling surge in the pre-midnight region. We count at least six clear pulses with a period of approximately one minute. Concurrent magnetic ground measurements show similar characteristics, almost simultaneously with the pulses in precipitation. We propose several possible mechanism for the pulsations, amongst them the theory of modulated wave particle interaction first proposed by Coroniti and Kennel (1970).
\end{abstract}

Key words. Storm and substorms; energetic particles, precipitating; wave-particle interaction

\section{Introduction}

In substorm research, whenever the term pulsating aurora is used, it is most often used for describing the recovery phase morning side precipitation pulsations. This is a very commonly, observed feature and is well described in statistical studies, for example, by Royrvik and Davis (1977). Although these pulsations can take on many different forms, they are most often found as diffuse patches, pulsating with periods from less than $1 \mathrm{~s}$ to several tens of seconds. These patches are known to be pulsating over long periods of time, up to $1 \mathrm{~h}$ or more. Pulsating aurora is also observed during other substorm phases and in other local time regions (Royrvik and

Correspondence to: A. Åsnes

(asnes@fi.uib.no)
Davis, 1977), but due to the high occurrence rate of the recovery phase pulsations, most of the theories trying to explain pulsating aurora have been focusing on explaining characteristics of the recovery phase pulsations. In our case, as we have observations of pulsations in the substorm expansion phase many of the theories developed for the recovery phase pulsations might not be adequate, and so we have tried to use the theories that might be applicable for our observations and also looked for other mechanisms that could cause the pulsations we observe in the energetic electron precipitation.

Pulsating behaviour in geomagnetic substorms can be found in many different parameters, for example, in the magnetic field, particle precipitation, ELF/VLF waves and cosmic absorption, and in many cases it is natural to expect there to be a connection between pulsations in different parameters. The most common form of pulsations used in substorm research is the $\mathrm{Pi} 2$ pulsations, irregular magnetic pulsations defined to have periods of 40 to $150 \mathrm{~s}$, which are found almost everywhere on the ground. This class of magnetic pulsations is in particular used as an onset signature and for timing of substorm onsets. A review of Pi2 pulsations can be found in Olson (1999). As Pi2 pulsations are known to occur in connection with substorm onset, a substorm phase which has not commonly been associated with pulsating aurora, Pi2 has not often been tied to pulsations in the electron precipitation. Geomagnetic ULF pulsations, in general though, have been known to accompany pulsating aurora, often with similar periodicities. While some theories claim that the geomagnetic pulsations are directly connected to the mechanism causing the pulsating aurora, others see the magnetic pulsations as an effect of the pulsating aurora, e.g. Sato and Matsudo (1986). As the conductivity changes with the modulated particle precipitation, the strength of the auroral currents will also change with time, resulting in magnetic pulsations measured on the ground. 
One explanation for the magnetic Pi2 pulsations observed in connection with substorm onsets is named the transient response mechanism and may also result in a modulated electron precipitation, e.g. (Goertz and Boswell, 1979; Baumjohann and Glassmeier, 1984). At substorm onset part of the magnetospheric cross-tail current is diverged into the ionosphere, forming the substorm current wedge. According to the transient response model part of this current, which travels to the ionosphere in the form of a kinetic Alfvén wave, is reflected and bounce back to the magnetosphere. The Pi2 signal is in turn a result of this current being turned on and off as the waves bounces back and forth between the ionosphere and the magnetospheric equatorial plane. Rothwell et al. (1986) included a more dynamic ionosphere into this model effecting the field-aligned current and pulsations, resulting in a more rapid damping in the low conductivity nightside ionosphere, but the general description still hold. According to the model of Rothwell et al. (1986) the field-aligned current is carried by electrons with typical energy of around $100 \mathrm{eV}$, but more energetic electrons will also be accelerated by more than $1 \mathrm{keV}$ by the electric field in the travelling wavefront.

The effects of particle precipitation on auroral currents was the motivation for Maehlum and O'Brien (1968) to propose an early model for pulsating aurora. Following a sudden increase in particle precipitation the strength of the ionospheric current should increase, leading to an increased magnetic field strength in the region poleward of the current. This increase will in turn make more particles mirror above the atmosphere, again reducing the conductivity and ionospheric current in this region. Maehlum and O'Brien did some calculations on this effect and found that it could lead to pulsations in the electron precipitation with a period from 50-300 s during periods of high activity.

The theory of Coroniti and Kennel (1970), on the other hand, claims that the magnetic ULF pulsations are the cause of the auroral pulsations. The Coroniti-Kennel, hereforth known as the CK-theory, is based on the findings of Kennel and Petschek (1966) that plasma with particle fluxes above a certain limit will give rise to growth of whistler waves resulting in rapid pitch angle diffusion and causing the particles to no longer be stably trapped. According to the CK-theory, there are certain plasma regimes where the mechanism of Kennel and Petschek (1966) will be sensitive to magnetic pulsations, resulting in time varying diffusion and hence particle precipitation. ULF pulsation waves can change the magnetic field strength, the anisotropy of the plasma and the plasma density, all of which will change the wave growth rate for the whistler mode. The CK-theory was later modified by Haugstad (1975), but the general results were still found to be valid. Of event studies supporting this theory is Glassmeier et al. (1988), who found a correlation between ground ULF pulsations and ELF wave intensity modulations. The main objective against the CK-theory has been that relevant magnetic pulsations have rarely been observed in the magnetosphere in conjunction with pulsating particle precipitation (Oguti, 1986).
Recently, Kepko and Kivelson (1999, 2001) have proposed a theory explaining observations of $\mathrm{Pi} 2$ pulsations in association with substorm onset. The theory explaines low latitude pulsations as the result of compressional pulses from the braking of earthward flowing bursty bulk flows. In several cases the pulse-shape was found to be similar in ground magnetic field measurements and earthward flow velocities in the near-Earth magnetospheric nightside. In addition, they proposed an additional mechanism for high latitude Pi2 pulsations through the same periodic braking of plasma in the near-Earth tail. This braking has theoretically been shown to produce an inertial current which should be a periodic addition to the substorm current wedge. We will also discuss the possibility for such an intermittent braking of bursty bulk flow, to give rise to pulsations in the electron precipitation rate.

\section{Instrumentation}

Our primary data source for this study are X-ray images from the Polar Ionospheric X-ray Imager Experiment (PIXIE) camera on the Polar satellite (Imhof et al., 1995). PIXIE is a multiple pinhole X-ray imager with a front and rear gas chamber detector. The front chamber is sensitive to $2-8 \mathrm{keV}$ photons, while the rear chamber detects $8-20 \mathrm{keV}$ photons. Polar was launched into a highly elliptical orbit with apogee over the Northern Hemisphere, but since then the positions of the apogee has gradually shifted towards the magnetospheric equatorial plane. For the event we are looking at, 24 July 1998, apogee was still almost directly over the Northern Hemisphere, while perigee, at a distance of $1.8-2.2 \mathrm{R}_{E}$, was over Antarctica. This results in a fairly high resolution during the perigee part of the orbit, with a spatial resolution of the order of $150 \mathrm{~km}$, and a temporal resolution of $10 \mathrm{sec}-$ onds. Due to its larger FOV, PIXIE is the only imager on Polar that provides global images from perigee. While the spatial resolution is not as high as for the imagers in visible and UV wavelengths, the temporal resolution of $10 \mathrm{~s}$ is actually better than that obtained from the other imagers on the Polar satellites, VIS (Frank et al., 1995) and UVI (Torr et al., 1995). One complete Polar orbit takes about $18 \mathrm{~h}$, but most of this time the satellite is hanging over the Northern Hemisphere. During the passage over the Southern Hemisphere, PIXIE is turned on for only $\sim 20 \mathrm{~min}$, as the camera has to be turned off while in the radiation belt to protect the electronics. To produce images with a sufficiently high signal-to-noise ratio, one has to integrate measured photon fluxes for a period of time, but during the Southern Hemisphere pass this would produce a smearing of the image as the satellite and the image field of view (FOV) moves during the image integration. To remove this effect a routine has been developed to use shorter integration intervals, such that the image movement is less than the spatial resolution of the image and then by superposing several images. The method used for superposing the images is to bin the images in a fixed grid in corrected geomagnetic (cgm) coordinates 
and magnetic local time (MLT). This method is possible for the PIXIE data because information on every photon measured by PIXIE is telemetered to the ground with energy and position, giving high flexibility for the image processing.

\section{Observations and interpretation}

During a Polar perigee pass on 24 July 1998, PIXIE imaged the late growth phase, substorm onset and expansion of a relatively intense substorm. The substorm we are investigating in this paper had an onset at 03:22 UT on 24 July 1998. From the $D_{s t}$ index we find that this substorm occurred in the late recovery phase of a weak geomagnetic storm. The $D_{s t}$ reached a minimum of $-48 \mathrm{nT}$ on the previous day, but had relaxed to $-24 \mathrm{nT}$ at the time of this substorm. Following some disturbances the previous day the auroral electrojet $(A E)$ index (Fig. 1) indicated some geomagnetic activity previous to the substorm, but this activity was declining and for about half an hour prior to the substorm the disturbance as measured by $A E$ was relaxed and reached a value less than $100 \mathrm{nT}$. Following substorm onset $A E$ rapidly reached a value of $1000 \mathrm{nT}$, indicating that this was a relatively intense substorm. From the $A E$ index we can also see several later intensifications. However, in this study we will focus on the first intensification, what we will refer to as the substorm onset and expansion phase, as this is the time period imaged by PIXIE (03:18-03:38 UT).

\subsection{Solar wind}

During this substorm Geotail was positioned in the solar wind directly in front of the magnetosphere close to the SunEarth line (GSE $(28.1,3.9,3.0))$ measuring, solar wind velocity and magnetic field as shown in Fig. 2. The magnetic field shows a period of southward interplanetary magnetic field (IMF) from around 03:00 to 03:18 UT when it returns to a northward direction. The northward turning of the IMF could be what triggers the substorm onset (Lyons, 1995). The period of southward IMF in this case is fairly short for a substorm and we notice that the magnitude of the southward component is only about $3 \mathrm{nT}$. However, we observe that the wind velocity is high, around $700 \mathrm{~km} / \mathrm{sec}$, which gives an efficient feeding of energy into the magnetosphere. Also, since this substorm occurs in the recovery phase of a weak magnetospheric storm, it is possible that the magnetosphere was already in a nearly unstable state even before the substorm growth phase started.

\subsection{Magnetospheric signatures}

In Fig. 4, panel (a), we show the magnetic $\mathrm{H}$ component of three stations at auroral latitudes. The location of these stations are shown in Fig. 3, panel A. The typical magnetic bay which is commonly observed in association with substorms is observed both at Poste de la Baleine, which is slightly to the west of the onset region, and further westward at Gillam, where the bay also starts very close to the onset time. At

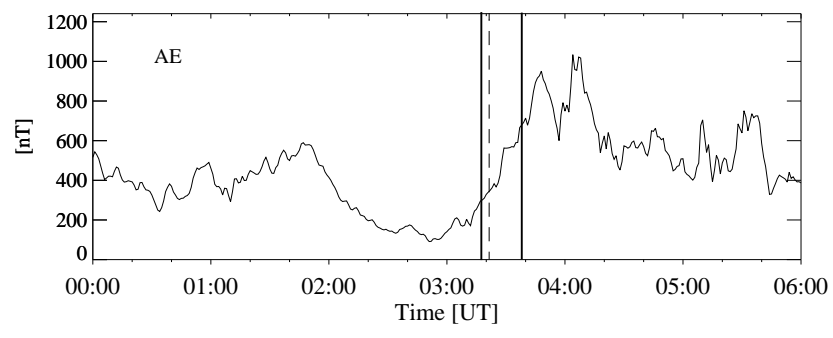

Fig. 1. Provisional AE index from Kyoto for 24 July, 1998. The two vertical lines indicate the time period of PIXIE images, the dashed line gives the time for the substorm onset.

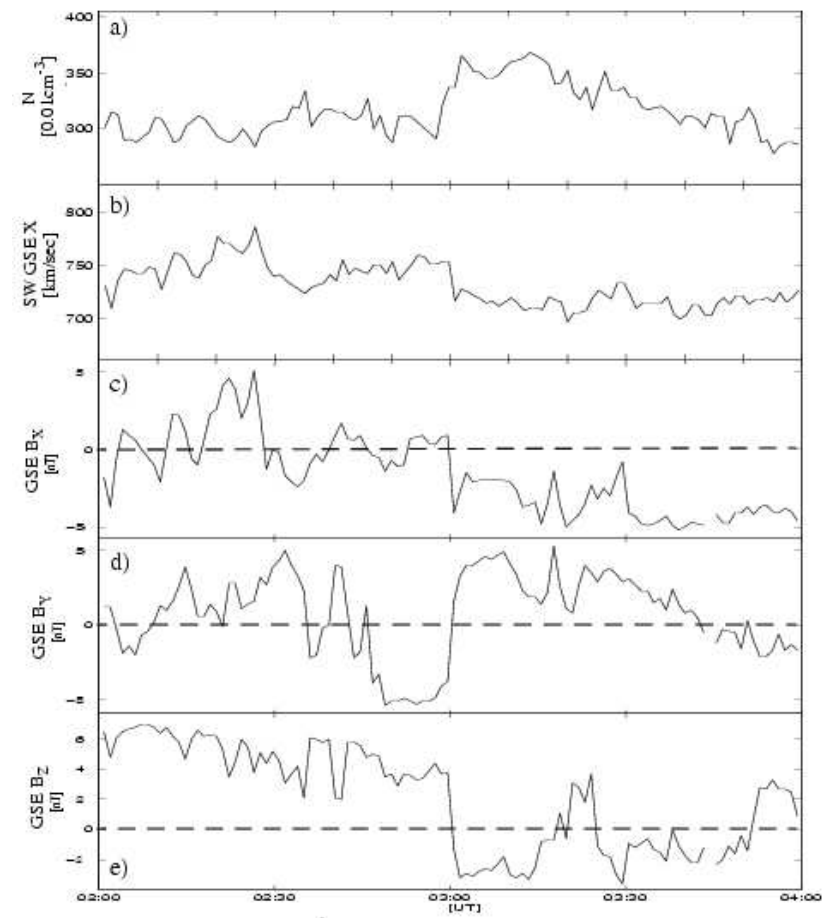

Fig. 2. Solar wind measurements by Geotail, 24 July 1998. From top to bottom the panels shows (a) particle density, (b) solar wind velocity in the GSM $x$-direction, and three components of the interplanetary magnetic field, (c) $B_{x}$, (d) $B_{y}$ and (e) $B_{z}$. The coordinate system is GSE.

Halley Bay, which is east of the onset region, the bay is relatively weak, which suggests that this station is outside of the substorm current wedge. The bottom panel shows the dipolarization observed by GOES-8 at geosynchronous orbit, around 22.20 MLT, west of the onset region. Here, the dipolarization follows the onset by $\sim 3 \mathrm{~min}$, which indicates that the satellite is initially outside of the current wedge but ends up inside the wedge as the wedge expands azimuthally in the magnetospheric equatorial plane.

Figure 3 shows a sequence of PIXIE images from the substorm. In the images the position of the ground stations are marked with small squares, the black are for the Northern Hemisphere and the red for Southern Hemisphere stations. The magnetic footprint of GOES- 8 mapped to the 


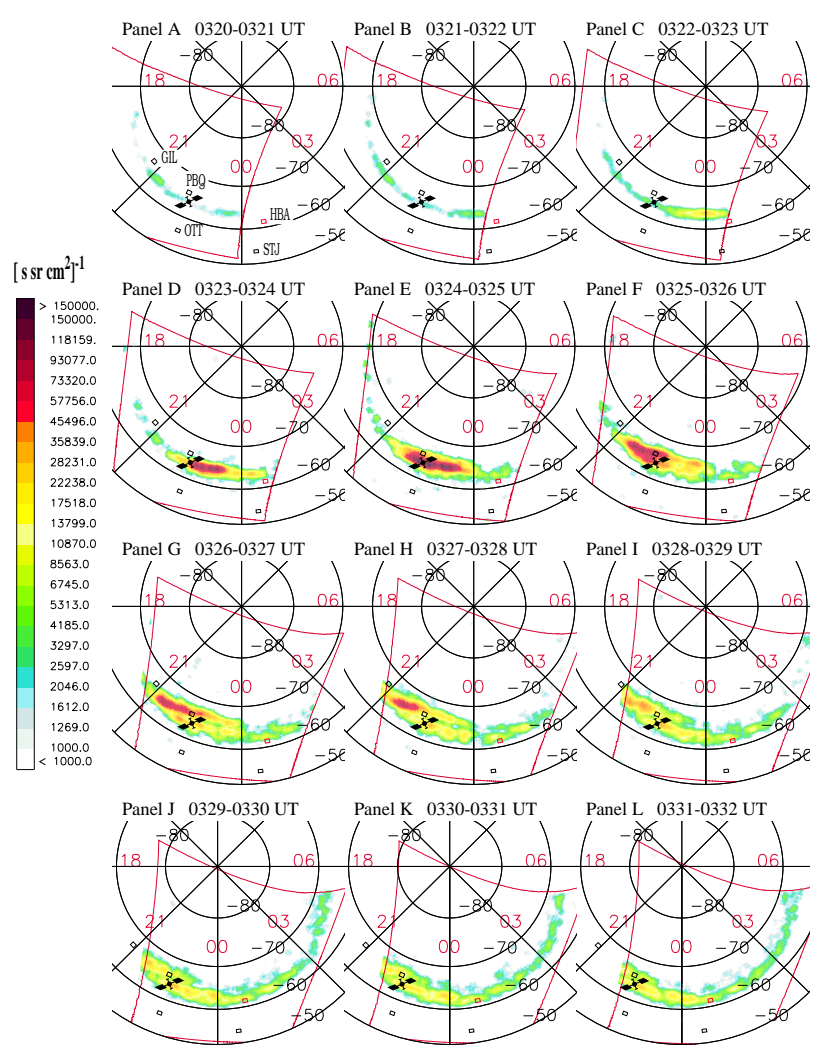

Fig. 3. PIXIE images from the substorm, 24 July 1998, energy range from $2.42-8.82 \mathrm{keV}$ photons. The coordinate system is corrected geomagnetic coordinates (cgm). Local midnight is straight downwards. The ground stations are marked in panel (a) as follows: GIL: Gillam, PBQ: Poste de la Baleine, HBA: Halley Bay, STJ: St. Johns, OTT: Ottawa. Halley Bay is the only Southern Hemisphere station. The magnetic footprint of GOES-8 is marked in black.

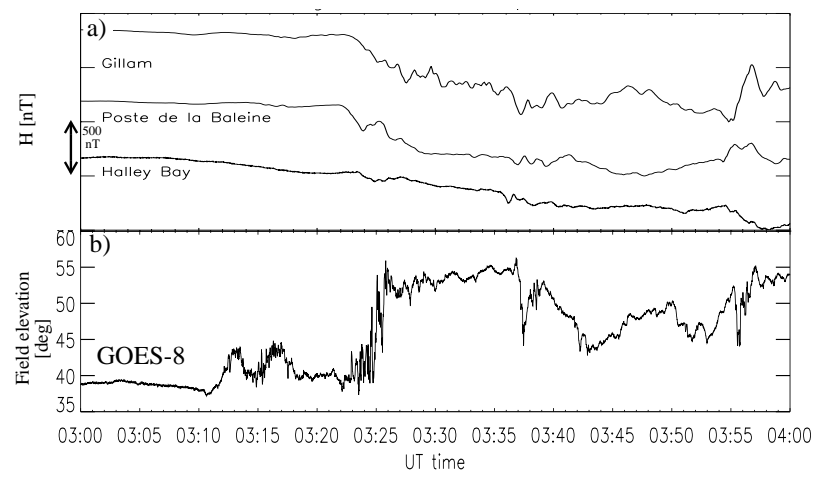

Fig. 4. Magnetic field measurements from different regimes, 24 July 1998. Panel (a) show magnetic H components from the ground stations Halley Bay, Poste de la Baleine and Gillam. Panel (b) show the magnetic field elevation measured in geosynchronous orbit by GOES-8 at 22:20 LT.

ionosphere by field line tracing through the Tsyganenko96 model (Tsyganenko, 1995) is also marked. The red line indicates PIXIE's field of view (FOV), while red numbers give

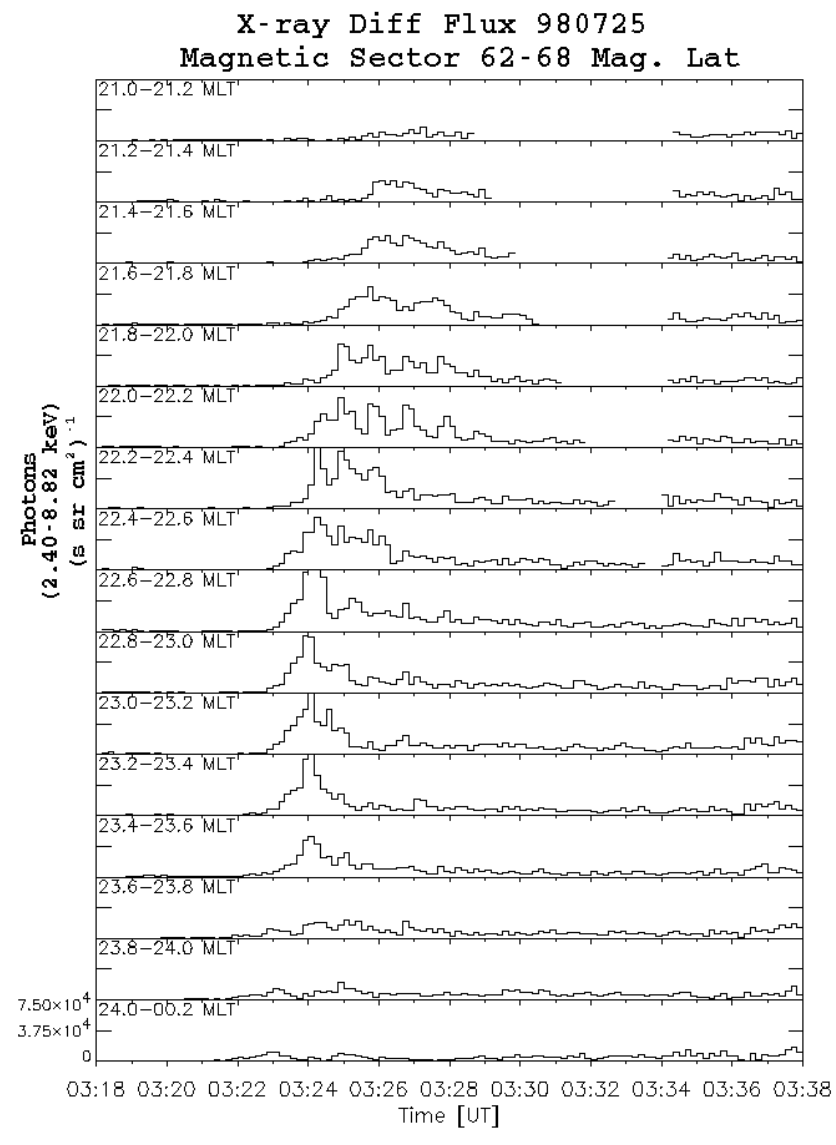

Fig. 5. Time development of X-ray fluxes (2.40-8.82 keV) for several MLT sectors. Fluxes are integrated for $10 \mathrm{~s}$, from $62^{\circ}-68^{\circ}$ latitude which covers the whole latitudinal range of the activity.

magnetic local time. Images are integrated for one minute. Panels (a) and (b) are from the growth phase, with a quiet arc stretching across the midnight sector within the FOV of PIXIE. This is relatively soft precipitation which cannot be seen in the PIXIE images at higher energies (not shown). In panel (c) the substorm onset is seen to occur in the magnetic midnight region, but in panel, (d), the area of maximum intensity has moved over to the pre-midnight sector, centered around 23.00 MLT. In the next panels the active region is expanding rapidly westward, reaching our limit of FOV close to 21.00 MLT in panel (h) at 03:27-03:28 UT. We also notice that from panel (f) and onwards the arc in the evening sector seems to split into two arcs, with the maximum intensity generally in the poleward arc.

\subsection{Pulsations in energetic electron precipitation}

In Fig. 5 we present the time development of the measured $\mathrm{X}$-ray fluxes. The X-ray fluxes are integrated for $10 \mathrm{~s}$ in areas of $6^{\circ}$ magnetic latitude (62-68) and 0.2 MLT in the local time regions, from the substorm onset region to the westward edge of the PIXIE's FOV. It is possible to use a better time resolution here than in the images because the integration areas are larger, resulting in higher count rates and better statistics. We 
notice that with the time resolution of ten seconds we have an ambiguity when we want to determine the exact time of substorm onset. The first activity that rises above a background level starts at 03:21:50 UT, with a maximum in the sectors around midnight MLT, but only a minute afterwards, at 03:22:50 UT, there is a rapid increase in activity centered around 23.00 MLT. This new activity is reaching fluxes more than five times higher than the previous activity in the midnight sector, where the activity is clearly declining when this new activity starts. For the remainder of this paper we will refer to the first activation as the substorm onset, although it should be remembered that the most intense activity begins one minute later. Also, it is not obvious that the onset time, as determined from the energetic electron precipitation, coincides with other features used for timing substorm onsets, for example, Pi2, visible auroral breakup, particle injection at geosynchronous orbit, dipolarization, bursty bulk flows (Liou et al., 1999). A striking feature in Fig. 5 is the modulation of fluxes in the local time sectors from 21.80 22.40 MLT. From the 22.20-22.40 MLT sector we observe that the pulsations initiate when the WTS reaches this MLT sector, resulting in three pulses in this sector. In the next sector (22.00-22.20 MLT) the activity arrives slightly afterwards, giving a first amputated pulse with a peak ten seconds after the peak of the first pulse in sector 22.20-22.40 MLT. The first pulse in the sector 21.80-22.00 MLT coincides with the second pulse in the two previous sectors, and they all reach the peak value simultaneously (within our time resolution of $10 \mathrm{~s}$ ). From this figure we can also estimate the degree of modulation from the height of the pulses. It follows that the modulation in at least some cases is more than $1: 1$ of the background unmodulated precipitation.

\section{$3.4 \quad$ PIXIE images}

To investigate the time development of the latitudinal profile of the pulsations we have plotted the PIXIE data as keograms for a selection of MLT sectors in Fig. 6. As in Fig. 5 the pulsations are visible in the three MLT sectors from 21.8022.40 MLT (Panel (b), (c) and (d)). From all the panels the poleward movement of the activity is obvious, and in some of the sectors a branching of the activity into one poleward and one equatorward branch can be seen. The three pulses in panel (d) are not very clear, mostly because of the logarithmic colour scale. In panel (c) it is easier to follow the evolution of the pulsations, through six pulses. The first three seem to cover the whole latitudinal range of the active area, while the following three pulses are stronger in the poleward part of the activity. This is also seen in panel B where the pulses again follow the poleward part of the activity.

\subsection{Magnetic field at geosynchronous orbit}

A satellite in geosynchronous orbit, GOES-8, is positioned in local time (22:40 LT), close to where we observe the pulsating particle precipitation. From a field line tracing by the Tsyganenko 95 model (Tsyganenko, 1995) we find that
GOES-8 is slightly equatorward and to the midnight side of the pulsations in the sector 22.20-22.40 MLT (see Fig. 3, panel (f). The magnetic field measurement of GOES- 8 is shown in the top three panels of Fig. 7 transformed into a field-aligned coordinate system. The top panel is along a 5-min averaged field direction, while $B_{y}$ and $B_{z}$ are perpendicular to the average field direction. The dashed lines indicates the start time of the most obvious pulses in the electron precipitation. From panel (a) we notice three clear compressional pulses, starting slightly before we see the first pulse in the particle precipitation. This corresponds well to the three pulses we see in the local time region of the GOES-8 footprint, in 22.20-22.40 MLT. In the two transversal directions in panel (b) and (c) we see some higher frequency pulsations, but the effect of a strongly modulated field-aligned current is hard to spot. In this case, where the satellite is earthward and slightly to the east of the westward leg of the substorm current wedge one would expect an increase in the field-aligned current to cause a negative excursion in the Y-component and possibly a small, positive effect in the Z-component. We have some possible explanations for this observation:

1) The modulation in precipitation is only effective for high energy electrons which constitutes a minor part of the field-aligned current; 2 ) The modulation of the current is very localised and hence the effect of the modulation at the position of GOES-8 drowns in the larger region of the current which is not modulated; 3) The magnetic field at GOES8 is very disturbed which could also hide any effects of a modulated FAC; 4) The mapping of field lines through the Tsyganenko model is wrong, and GOES- 8 is too far away from the modulated field-aligned current to observe modulations in the magnetic field. As GOES-8 is on the equatorward side of the downward leg of the substorm current wedge, it is likely that this current to some extent twists the magnetic field. The effect should be that the footpoint of GOES-8 is in reality closer to midnight than what we obtained from the mapping by the Tsyganenko model, being further away from the field-aligned current.

\subsection{Geomagnetic waves at the ground}

For this substorm there were no Southern Hemisphere ground stations close to the area of electron precipitation pulsations, but most likely this phenomenon occurred in both hemispheres and so we would like to look for magnetic pulsations in the Northern Hemisphere. Panel (g) of Fig. 7 shows the H-component of the magnetic field at the two subauroral stations of Ottawa and St. Johns. Both stations show Pi2-pulsations in association with the substorm, both in the $\mathrm{H}$ and $\mathrm{D}$ (not shown) components. Comparing the pulses in electron precipitation and magnetic field we notice that pulsations at Ottawa, which is in the local time of the electron pulsations, seem to start half a period previous to the peak in particle precipitation. Further, the precipitation peaks seem to correlate well with the minimum of the magnetic pulsations. At St. Johns, which is directly eastward of the onset region, close to local midnight, the pulsations are slightly 
Panel A 21.6-21.8 MLT

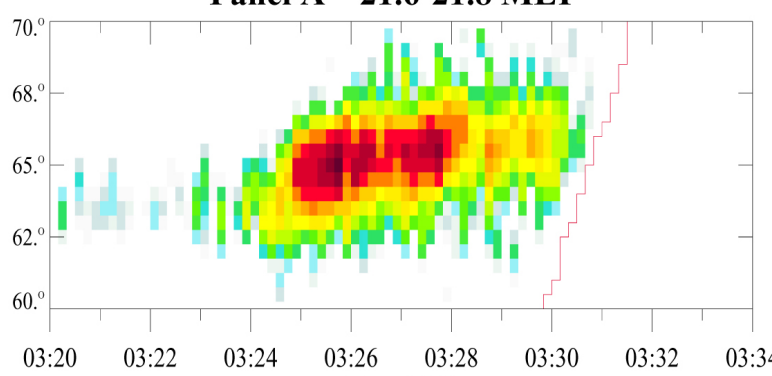

Panel C 22.0-22.2 MLT

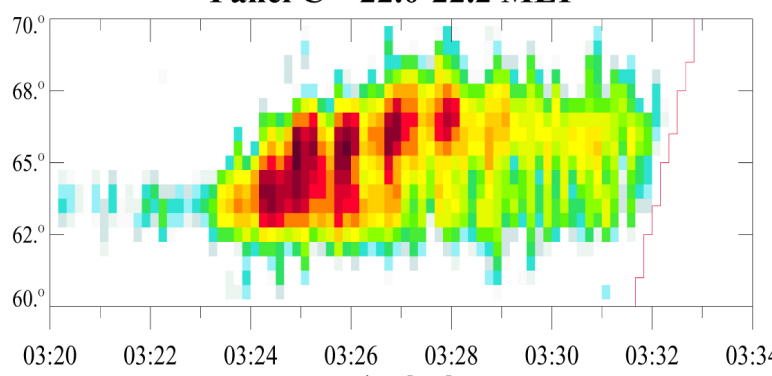

Panel E 22.4-22.6 MLT

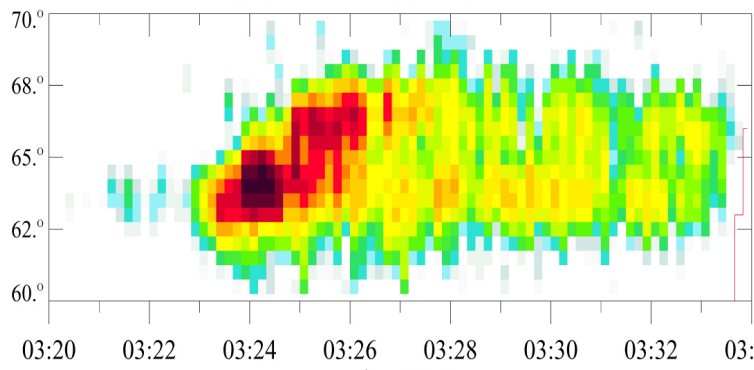

Panel G 22.8-23.0 MLT

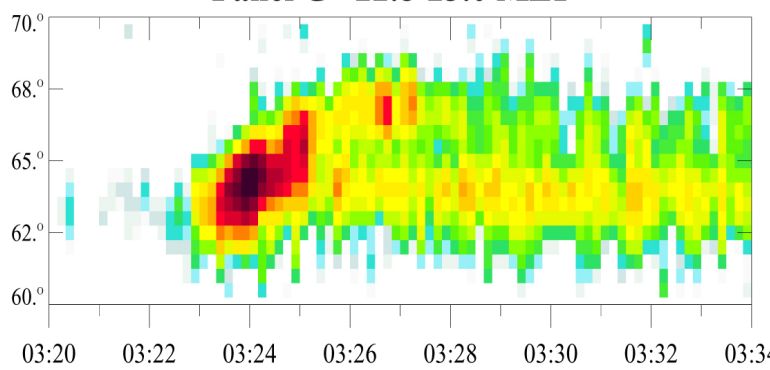

Panel B 21.8-22.0 MLT

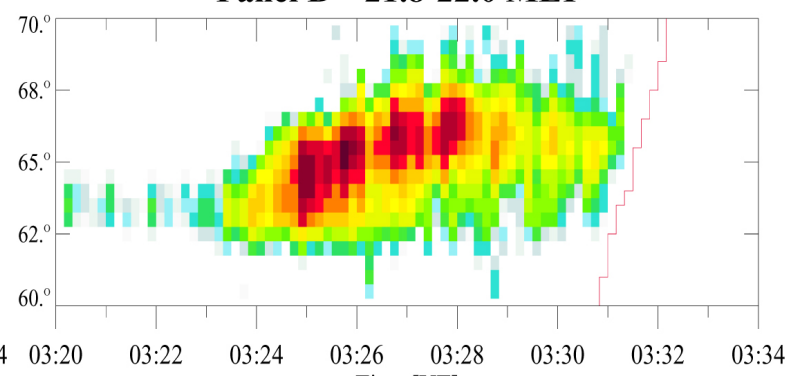

Panel D 22.2-22.4 MLT

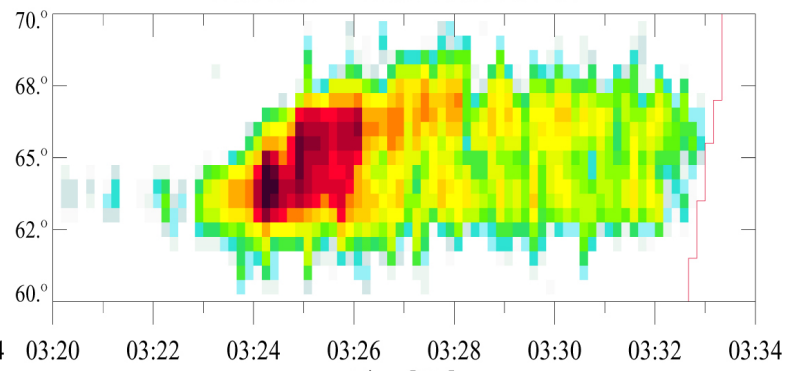

Panel F 22.6-22.8 MLT

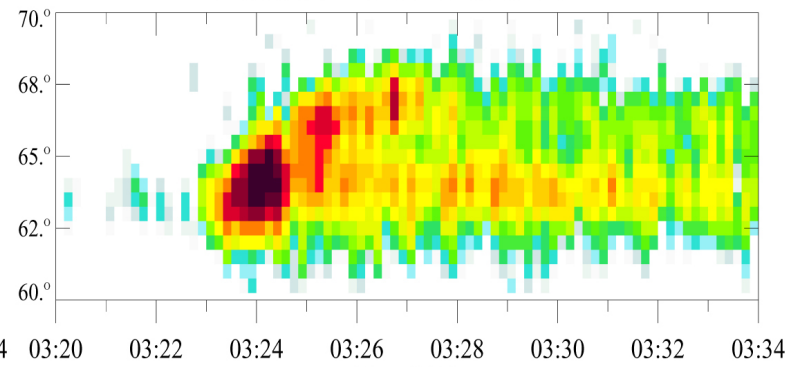

Panel H 23.6-23.8 MLT

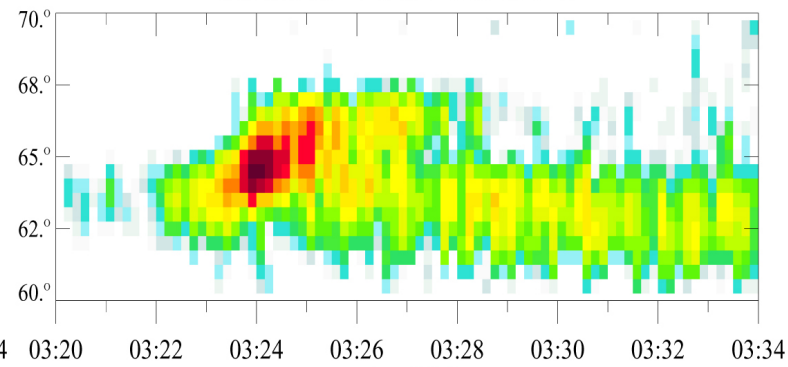

Fig. 6. Keograms for a selection of MLT sectors produced from $2.40-8.82 \mathrm{keV}$ photon measurements by PIXIE. The colour scale is the same as in Fig. 3 and the red line give the border of FOV.

delayed with respect to Ottawa. Although St. Johns is closer than Ottawa to the first weak onset region, it is further away from the region of main intensity around 23.00 MLT. Therefore, it is not surprising that the pulsations which are believed to originate from the surge arrives at Ottawa first.

\section{Discussion}

In general, there are at least two ways one can obtain pulsations in the energetic electron precipitation. One is through a modulation in the mechanism responsible for the injection of energetic electrons at substorm onset and the other is a modulation in the precipitation rate of the already injected electrons. For the pulsations we are investigating in this paper the injection of new particles is clearly an ongoing process at the time of the pulsations since the active area is still expanding towards the evening side, in the opposite direction of the electron drift. The particle precipitation modulations could in this case be due to a modulated injection process but the physics is obviously dependent on the injection mechanism itself, an issue which has not yet been solved. 


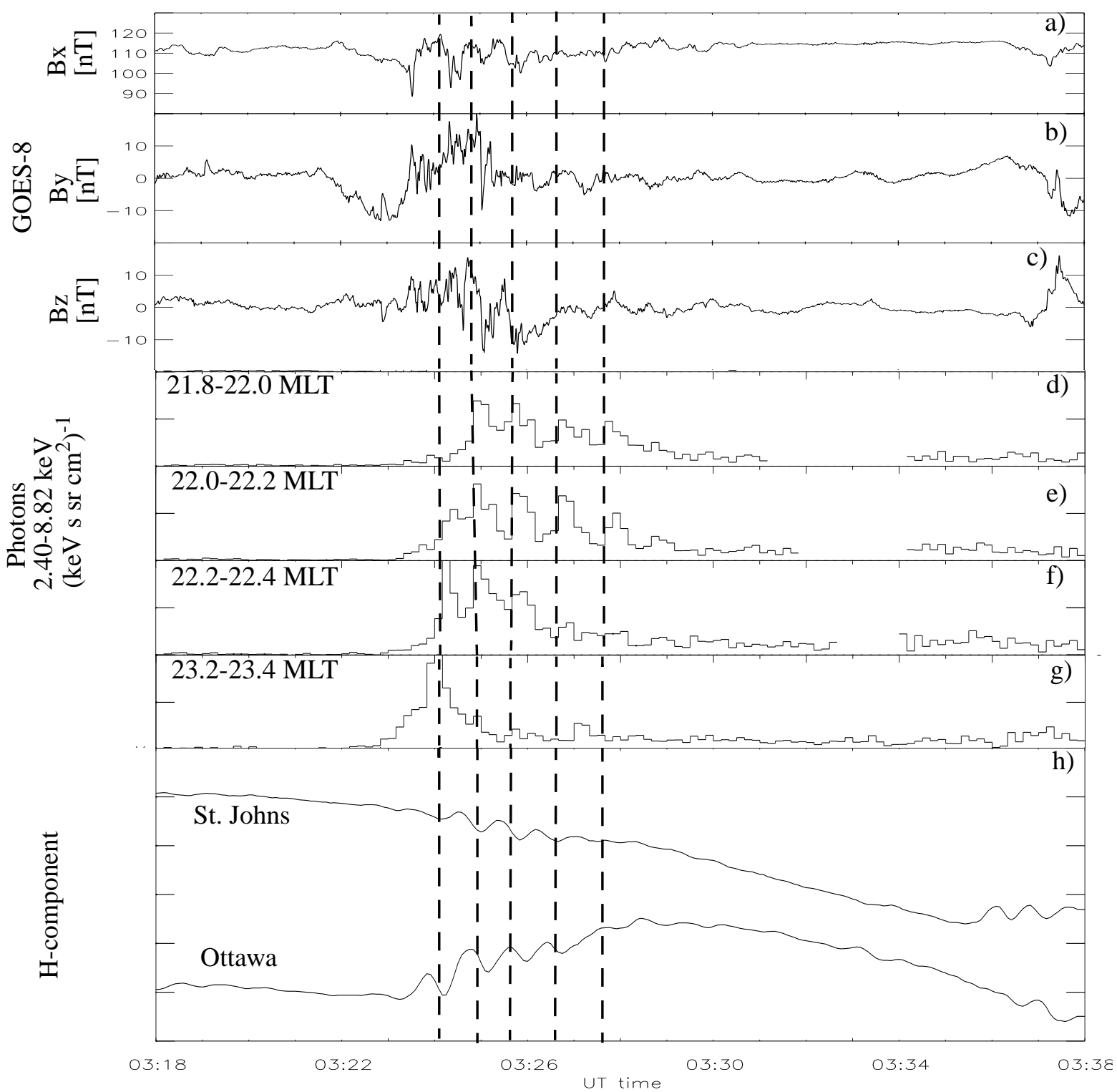

Fig. 7. A composite plot. Panels (a) to (c) show the magnetic field measured by GOES-8 in geosynchronous orbit converted into fieldaligned coordinates. The field-aligned direction, $B_{x}$, is based on a $5 \mathrm{~min}$ average field direction. $B_{y}$ is in the direction perpendicular to $B_{x}$ and the spin aligned component $B_{p}$ which is northward. Panels (d),(e) and (f) are selected MLT sectors from Fig. 5. Panel (g) shows the H component of the two Northern Hemisphere stations Ottawa and St. Johns.

Several instabilities have been proposed for the onset near geosynchronous orbit (e.g. Erickson et al., 2000; Lui, 1996), while an equally popular theory starts out with an instability further tailward, causing a near-Earth neutral line (Shiokawa et al., 1998). In the framework of this latter theory the pulsations could be due to a localised periodical flow braking (Kepko and Kivelson, 2001). The mechanism for the pulsations, an effect of localised braking of a periodic bursty bulk flow is a part of what is currently the most popular nearEarth neutral line model (e.g. Haerendel, 1992; Shiokawa et al., 1998). In this model the near-Earth reconnection accelerates plasma towards the Earth which is subsequentially slowed down and finally stops when it arrives at the more dipolar magnetic field in the near geosynchronous region. Bursty bulk flows have been shown to be the dominant mechanism of plasma transport in the plasma sheet and plasma sheet boundary layer (Angelopoulos et al., 1994). Kepko and Kivelson (2001) has previously found evidence that periodicities in the velocity variations in a bursty bulk flow results in similar wave forms in the magnetic field at ground stations and we propose that such a mechanism could also result in periodicities in the particle precipitation. A braking of bursty bulk flows could be imagined to give a periodical, localised injection of new particles with the same periodicity as what one would observe with a satellite in the bulk flow. However, in our case we have no observations in the magnetosphere to 
support such a view. In general, one would have to be fortuitous to observe the earthward bursty bulk flows and simultaneously image the aurora with a time resolution high enough to resolve the waveform of modulations in the particle precipitation. However, as Kepko found a similarity between waveforms in the ground magnetic field and bursty bulk flow velocities, it should be possible to find similarities in particle precipitation and magnetic field, if they have the same origin. Most of the events studied by Kepko and Kivelson (2001) were substorms following a quiet period. Only during such periods were they able to find the similarities in the waveform because any disturbances previous to the substorm tended to disturb the magnetic field enough to hide the effects of the braking of the BBF. This might be an explanation of why we have not been able to find any good correlation between waveforms in particle precipitation and magnetic field measurements on mid latitudes at dusk and dawn (not shown).

One theory that could explain the pulsations through a modulation of the precipitation rate is the theory of Coroniti and Kennel (1970) with a wave-particle interaction modulated by ULF-waves. According to the theory of Coroniti and Kennel (1970), it is possible to obtain modulations of energetic electron precipitation from magnetic ULF pulsations. The theory assumes an initially stable interaction between VLF waves and particles which is destabilised by the geomagnetic pulsations. The wave-particle interaction is thought to take place in the magnetic equatorial plane, and this would also be the source region of the modulation of precipitating electrons. In this situation one would expect to see magnetic pulsations following the precipitation modulations due to the difference in travel time of electrons and magnetic waves. The electrons will go from the magnetospheric equatorial plane to the ionosphere on a time scale of seconds, while magnetic waves will spend a time of the order of a minute, depending on the plasma properties and the tailward distance of the location of modulation. From Fig. 7 we can see that the geomagnetic pulsations arrive simultaneously or even previously to the modulations in the particle precipitation. This speaks against the Coroniti-Kennel mechanism, but there could be explanations. At the local time where we observe the pulsations there was no precipitation previous to when the pulsations start. It is, therefore, possible that the magnetic pulsations already existed in the magnetosphere before the injection of energetic electrons arrived at this local time, and only then started to modulate the wave particle interaction. In this case the magnetic pulsations could be expected to be found simultaneously or even previous to the modulation in particle precipitation. One could even imagine that the magnetic pulsations that cause the modulation in particle precipitation was initiated at the substorm onset, possibly in the ionosphere, and only later reached the magnetospheric equatorial plane, where it could start to modulate the precipitating particles. There is also a possibility that the waves modulating the wave particle precipitation are standing non-propagating waves. This would also explain why the magnetic pulsations are observed simultaneously with the pulsating precipitation. For this sub- storm we have available magnetic field measurement from the geosynchronous region close to the region of pulsating electron precipitation. From the Coroniti-Kennel theory one would expect this field to show ULF pulsations of a similar frequency as the pulsating precipitation. Such pulsations are not seen in this case, but an explanation for this could be that GOES-8 is only close but not inside the pulsating region, and not in a position to measure such ULF waves.

In general, one would expect the waves causing the pitch angle diffusion of energetic electrons into the loss cone to be of whistler wave mode. However, the whistler wave mode has to satisfy the limitation $\omega<\Omega_{c e}$, where $\omega$ is the wave frequency and $\Omega_{c e}$ is the electron gyrofrequency. If we assume that the magnetic field strength is similar to or lower than that measured at this time close to the observed pulsations by GOES- 8 , we find that the waves are limited to frequencies below $3 \mathrm{kHz}$ (ELF frequencies). Also, an approximation to the resonance condition for parallel-propagating waves where $\omega_{p} \gg \omega$ is given by Eq. (1) (Kennel and Petschek, 1966). Here, $\omega_{p}$ is the electron plasma frequency, $B$ is the local magnetic field strength, $n_{e}$ is the cold electron density and $\mu_{0}$ is the vacuum permeability. Calculating the first term on the right-hand side with $B=100 \mathrm{nT}$ and $n_{e}=1.0 \mathrm{~cm}^{3}$ results in a value of $\sim 25 \mathrm{keV}$. It has been common in the literature to use $\frac{\omega}{\Omega_{c e}}=0.5$ (Isenberg et al. 1982) and with this value the lower parallel resonance energy will be $\sim 12 \mathrm{keV}$. This shows that the wave-particle interaction in this case will diffuse electrons with energies relevant to what we measure with PIXIE

$E_{R \|}=\frac{B^{2}}{2 \mu_{0} n_{e}} \frac{\Omega_{c e}}{\omega}\left(1-\frac{\omega}{\Omega_{c e}}\right)^{3}$.

\section{Conclusion}

We have shown observations from multiple sources for a substorm occurring on 24 July 1998. The data set with the observations of pulsations of energetic electron precipitation in the Pi2 range has been thoroughly discussed within the limits of our observations, and different mechanisms for these pulsations were proposed. Unfortunately, we have so far found only this event in our data set that shows this feature. In this case we have several observations in relevant positions from the ionosphere and magnetosphere, but there are still many observations lacking. For example, it would have been clarifying with respect to the periodic flow braking theory to have observations of the bursty bulk flow (if this exists). Also, VLF observations on the ground or in the magnetosphere in the position to detect eventual wave growth in the source region for the pulsating electron pulsations would be necessary for discussing the theory of modulated wave-particle mechanism more thoroughly. 
Acknowledgements. We would like to thank WDC-Kyoto for providing the provisional $A E$ and the final $D_{s t}$ index. Thanks to $\mathrm{H}$. Singer at NOAA SEC for providing GOES-8 data and for useful comments on this paper. Thanks to Natural Resources Canada, Geological Survey of Canada, CANOPUS and British Antarctica Survey (BAS) for magnetic field data. Financial support for this study has been provided by the Research Council of Norway. The participation of the University of Maryland in this research was supported by Lockheed martin subcontract SE70A0470.

Topical Editor T. Pulkkinen thanks two referees for their help in evaluating this paper.

\section{References}

Angelopoulos, V., Kennel, C. F., Coroniti, F. V., Pellat, R. W., Kivelson, M. G., Walker, R. J., Russel, C., Baumjohann, J., Feldman, W., and Gosling, J.: Statistical characteristics of bursty bulk flow events, J. Geophys. Res., 99, 21 257, 1994.

Baumjohann, W. and Glassmeier, K-H.: The transient response mechanism and Pi2 pulsations at substorm onset - Review and outlook, Planet. Space Sci., 32, 1361-1370, 1984.

Coroniti, F. V. and Kennel, C. F.: Electron precipitation pulsations, J. Geophys. Res., 75, 1279, 1970.

Erickson, G. M., Maynard, N. C., Burke, W. J., Wilson, G. R., and Heinemann, M. A.: Electromagnetics of substorm onsets in the near-geosynchronous plasma sheet, J. Geophys. Res. 105, 25 265-25 290, 2000.

Frank, L. A., Sigwarth, J. B., Craven, J. D., Cravens, J. P., Dolan, J. S., Dvorsky, M. R., Hardebeck, P. K., Harvey, J. D., and Muller, D. W.: The visible imaging system (VIS) for the polar spacecraft, Space Sci. Rev., 71, 297-328, 1995.

Glassmeier, K.-H., Baumjohann, W., Korth, A., and Gough, P.: High-latitude Pi2 pulsations, ELF intensity, and particle flux variatinos: A case study, Ann. Geo., 6, 287-296, 1988.

Goertz, C. K. and Boswell, R. W.: Magnetosphere-ionosphere coupling, J. Geophys. Res., 84, 7239-7246, 1979.

Haerendel, G.: Disruption, ballooning or aurorlal avalanche - On the cause of substorms, Proc. ICS-1, Kiruna, 1992.

Haugstad, B. S.: Modification of a theory of electron precipitation pulsations, J. Atmos. Terr. Phys., 37, 257-272, 1975.

Imhof, W. L., Spear, K. A., Hamilton, J. W., Higgins, B. R., Murphy, M. J., Pronko, J. G., Vondrak, R. R., McKenzie, D. L., Rice, C. J., Gorney, D. J., Roux, D. A., Williams, R. L., Stein, J. A., Bjordal, J., Stadsnes, J., Njoten, K., Rosenberg, T. J., Lutz, L., and Detrick, D.: The polar ionospheric X-ray imaging experiment (PIXIE), Space Sci. Rev., 71, 385, 1995.

Isenberg, P. A., Koons, H. C., and Fennel, J. F.: Simultaneous observations of energetic electrons and dawnside chorus in geosynchronous orbit, J. Geophys. Res.,87, 1495-1503, 1982.

Kennel, C. F. and Petschek, H. E.: Limit on stably trapped particle fluxes, J. Geophys. Res., 71, 1, 1966.
Kepko, E. L. and Kivelson, M. G.: Generation of Pi2 pulsations by bursty bulk flows, J. Geophys. Res., 104, 25 021, 1999.

Kepko, E. L. and Kivelson, M. G.: Flow bursts, braking and Pi2 pulsations, J. Geophys. Res., 106, 1903-1915, 2001.

Liou, K., Meng, C. I., Lui, A. T. Y., Newell, P. T., Brittnacher, M., Parks, G., Reeves, G. D., Anderson, R. R., and Yumoto, K.: On relative timing in substorm onset signatures, J. Geophys. Res., 104, 22 807-22 817, 1999.

Lui, A. T. Y.: Current disruption in the earth's magnetosphere: Observations and models, J. Geophys. Res., 101, 13 067-13 088, 1996.

Lyons, L. R.: A new theroy for magnetospheric substorms, J. Geophys. Res., 100, 19069-19081, 1995.

Meng, C. -I., Mauk, B., and McIlwain, C. E.: Electron precipitation of evening diffuse aurora and its conjugate electron fluxes near the magnetospheric equator, J. Geophys. Res.,84, 2545, 1979.

Maehlum, B. N. and O'Brien, B. J., The mutual effect of precipitated auroral electrons and the auroral electrojet, J. Geophys. Res., 73, 1679-1684, 1968.

Oguti, T., Hayashi, K., Yamamoto, T., Ishida, J., Higuchi, T., and Nishitani, N.: Absence of hydromagnetic waves in the magnetospheric equatorial region conjugate with pulsating auroraa, J. Geophys. Res., 91, 13 711-13 715, 1986.

Olson, V. J.: Pi2 pulsations and substorm onsets: A review, J. Geophys. Res., 104, 17 499, 1999.

Paquette, J. A., Matthews, D. L., Rosenberg, T. J., Lanzerotti, L. J., and Inan, U. S.: Source regions of long-period pulsation events in electron precipitation and magnetic fields at South Pole Station, J. Geophys. Res., 99, 3869, 1994.

Rothwell, P. L., Silevitch, M. B., and Block, L. P.: Pi 2 Pulsations and the westward traveling surge, J. Geophys. Res., 91, 69216928, 1986.

Royrvik, O. and Davis, T. N.: Pulsating Aurora: Local and global morphology, J. Geophys. Res., 82, 4720, 1977.

Sato, N. and Kokubun, S.: Interactions between ELF-VLF emissions and magnetic pulsations: Quasi-periodic ELF-VLF emissions associated with Pc 3-4 magnetic pulsations and their geomagnetic conjugacy, J. Geophys. Res., 85, 101, 1980.

Sato, N. and Matsudo, T.: Origin of magnetic pulsations associated with regular VLF pulsations (type $2 \mathrm{QP}$ ) observed on the ground at Syowa Station, J. Geophys. Res., 91, 11 179-11 185, 1986.

Shiokawa, K., Baumjohann, W., Haerendel, G., Pashmann, G., Fennell, J. F. Friis-Christensen, E., Lühr, H., Reeves, G. D., Russel, C. T., Sutcliffe, P. R., and Takahashi, K.: High-speed ion flow, substorm current wedge, and multiple Pi2 pulsations, J. Geophys. Res., 102, 4491-4507, 1998.

Torr, M. R., Torr, D. G., Yukic, M., Johnson, R. B., Ajello, J., Banks, P., Clark, K., cole, K., Keffer, C., Parks, G., Tsurutani, B. and Spann, J.: A far ultaviolet imager for the international solar-terrestrial physics mission, Space Sci. Rev., 71, 329, 1995.

Tsyganenko, N. A.: Modelling the earth's magnetospheric magnetic field confined within a realistic magnetopause, J. Geophys. Res., 100, 5599, 1995. 